\title{
Análise da distribuição espacial e evolução do número de áreas contaminadas nas
}

\section{bacias hidrográficas dos rios Aguapeí e Peixe}

\author{
Analysis of the spatial distribution and evolution of the number of contaminated areas in the \\ hydrographic basins of the Aguapeí and Peixe Rivers
}

Análisis de la distribución espacial y evolución del número de áreas contaminadas en las cuencas

hidrográficas de los ríos Aguapeí y Peixe

Recebido: 16/06/2021 | Revisado: 21/06/2021 | Aceito: 22/06/2021 | Publicado: 09/07/2021

\author{
Osmar Toledo Bonfim \\ ORCID: https://orcid.org/0000-0001-8863-8313 \\ Universidade Federal de Santa Maria, Brasil \\ E-mail: osmartoledob@gmail.com \\ Denilson Burkert \\ ORCID: https://orcid.org/0000-0002-8451-7562 \\ APTA - Polo Regional Alta Paulista, Brasil \\ E-mail: denilson.burkert@sp.gov.br \\ Mariana Ribeiro Fernandes \\ ORCID: https://orcid.org/0000-0002-4471-9485 \\ Universidade Estadual Paulista Júlio de Mesquita Filho, Brasil \\ E-mail: marianaribeiro.f06@gmail.com \\ Alan Lima dos Reis \\ ORCID: https://orcid.org/0000-0002-7214-6119 \\ Instituto Tecnológico de Aeronáutica, Brasil \\ E-mail: al.reis2012@hotmail.com
}

\begin{abstract}
Resumo
Área contaminada é definida como área, terreno, local, instalação ou edificação que contenha quantidades, ou concentrações de matéria, em condições que causem ou possam causar danos à saúde humana, meio ambiente ou a outro bem a proteger. Buscando obter um melhor entendimento a respeito das áreas contaminadas nas bacias hidrográficas do rio Aguapeí e do rio do Peixe (BHAP), o objetivo deste trabalho foi realizar o levantamento das áreas contaminadas nas BHAP, identificar as principais atividades potencialmente contaminantes e os meios físicos mais afetados, e simultaneamente, correlacionar os possíveis valores de contaminação com os parâmetros socioeconômicos dos respectivos municípios. Foram utilizados dados de áreas contaminadas no período de 2007 à 2019 dos municípios pertencentes às BHAP, obtidos através dos Relatórios de Áreas Contaminadas e Reabilitadas do Estado de São Paulo publicados pela Companhia Ambiental do Estado de São Paulo. Foram identificados 26 municípios com áreas contaminadas, sendo os postos de combustíveis a principal atividade contaminante, enquanto os solventes aromáticos e os hidrocarbonetos policíclicos aromáticos foram os contaminantes mais relatados. As águas subterrâneas e os subsolos foram, majoritariamente, os meios mais impactados pelos contaminantes. Uma correlação forte foi encontrada entre o maior número de contaminantes e as cidades com maior área e também com maior número de habitantes. De modo geral, a identificação dos locais contaminados em bacias hidrográficas configura-se como uma importante ferramenta que visa auxiliar no gerenciamento das áreas contaminadas e na adoção de ações mitigatórias e a produção de políticas públicas que visem amenizar os efeitos das contaminações.
\end{abstract}

Palavras-chave: Política pública; Gestão ambiental; Meio ambiente; Bacia hidrográfica; Contaminação.

\begin{abstract}
Contaminated site is defined as an area, land, location, installation or building that contains quantities or concentrations of matter, in conditions that cause or may cause damage to human health, the environment or other property to be protected. Seeking to obtain a better understanding of the contaminated sites in the hydrographic basins of the Aguapeí River and Peixe River (BHAP), the aim of this study was to survey the contaminated areas in the BHAP, identify the main potentially contaminating activities and the most affected physical environments, and simultaneously correlate the possible contamination values with the socioeconomic parameters of the respective cities. We selected contaminated sites data from cities belonging to the BHAP in the period from 2007 to 2019, obtained through the Contaminated and Rehabilitated Areas Reports of the State of São Paulo published by the Environmental Company of São Paulo State (CETESB). We identified 26 municipalities with contaminated sites, with gas stations
\end{abstract}


being the main activity responsible for the contamination, while aromatic solvents and polycyclic aromatic hydrocarbons (PAHs) were the most reported contaminants. Groundwater and subsoil were, for the most part, the environments most impacted by contaminants. A strong correlation was found between the largest number of contaminants and cities with the largest area and largest number of inhabitants. In general, the identification of contaminated sites in hydrographic basins is an important tool that aims to assist in the management of contaminated areas and in the adoption of mitigation actions and the production of public policies aimed at mitigating the effects of contamination.

Keywords: Public policy; Environmental management; Environment; Hydrographic basin; Contamination.

\begin{abstract}
Resumen
Área contaminada se define como un área, terreno, ubicación, instalación o edificio que contiene cantidades o concentraciones de materia, en condiciones que causan o pueden causar daños a la salud humana, el medio ambiente y otra propiedad a proteger. Buscando obtener un mejor conocimiento de las áreas contaminadas en las cuencas hidrográficas del río Aguapeí y del río Peixe (BHAP), el objetivo de este trabajo fue relevar las áreas contaminadas en las BHAP, identificar las principales actividades potencialmente contaminantes y los ambientes físicos más afectados, y simultáneamente correlacionar los posibles valores de contaminación con los parámetros socioeconómicos de los respectivos municipios. Se utilizaron datos de áreas contaminadas entre los años 2007 y 2019 de los municipios pertenecientes al BHAP, obtenidos a través de los Informes de Áreas Contaminadas y Rehabilitadas del Estado de São Paulo que son publicados por la Empresa Ambiental del Estado de São Paulo. Se identificaron 26 municipios con áreas contaminadas, siendo las gasolineras las principales responsables de la contaminación, mientras que los solventes aromáticos y los hidrocarburos aromáticos policíclicos fueron los contaminantes más reportados. El agua subterránea y el subsuelo fueron, en su mayor parte, los medios más afectados por los contaminantes. Se encontró una fuerte correlación entre la mayor cantidad de contaminantes y las ciudades con mayor superficie y también las de mayor población. En general, la identificación de sitios contaminados en cuencas hidrográficas es una herramienta importante que tiene como objetivo ayudar en el manejo de áreas contaminadas y en la adopción de acciones de mitigación y la elaboración de políticas públicas orientadas a mitigar los efectos de la contaminación.
\end{abstract}

Palabras clave: Políticas públicas; Gestión ambiental; Medio ambiente; Cuenca hidrográfica; Contaminación.

\title{
1. Introdução
}

A expansão desordenada dos centros urbanos tem sido sinônimo de degradação ambiental antropogênica, configurando como uma das maiores adversidades enfrentadas pela sociedade atualmente. Ao mesmo tempo a intensificação das atividades industriais, o uso e ocupação do solo e a exploração de recursos hídricos sem observar os parâmetros de proteção ambiental, aumentam o número de áreas contaminadas no espaço urbano (Zasso et al., 2014).

O conceito de áreas contaminadas não se limita apenas ao in loco da contaminação, em outras palavras, ao solo que recebeu os contaminantes, sendo assim, a área contaminada envolve o conjunto dos diferentes meios (ar, água, solo, vegetação) afetados. Nessas áreas, os poluentes e contaminantes podem se disseminar pela superfície do terreno, afetando outros ambientes como subsolo, águas superficiais e subterrâneas, ar, biota e também os ambientes construídos, como edificações e instalações, além de causar riscos à saúde humana e outros receptores (Günther, 2006; Panagos et al., 2013)

Segundo Sánchez (2001) existem quatro principais problemas decorrentes da contaminação de áreas, são: risco à saúde humana e aos ecossistemas; risco à segurança dos indivíduos e da propriedade; redução do valor imobiliário da propriedade; e restrições ao desenvolvimento urbano. Além desses, ainda ocorre a possibilidade de contaminação dos recursos hídricos, especialmente águas subterrâneas utilizadas para abastecimento público.

Ao longo do tempo pesquisas têm sido geradas buscando analisar a diversificação, intensificação e a espacialização de áreas contaminadas de origem antropogênica. Marques et al. (2007) avaliaram a presença de áreas contaminadas provenientes de atividades agrícolas; Pinheiro e Mochel (2018) identificaram áreas contaminadas por metais pesados; enquanto que Carneiro et al. (2020) verificaram inúmeras áreas contaminadas por compostos orgânicos no estado de Minas Gerais.

No Brasil, a contaminação dos recursos hídricos e do subsolo ocorre pelo resultado das atividades humanas. Em áreas de grande concentração populacional em que o uso da terra é intenso, o solo e as águas subterrâneas estão constantemente vulneráveis a um potencial poluição. Somente no estado de São Paulo, 80\% dos municípios utilizam as águas subterrâneas como recurso hídrico principal ou único para o abastecimento urbano (CETESB, 2012). 
A origem da determinação de áreas contaminadas está relacionada ao desconhecimento, no passado, dos procedimentos seguros para a manipulação de substâncias perigosas, ao desrespeito a esses métodos seguros e a episódios de acidentes ou de vazamentos durante o desenvolvimento dos processos produtivos, seja no transporte ou no armazenamento de matérias primas e produtos (CETESB, 2015; Braun et al., 2020). Segundo Hirata e Ferreira (2001) as atividades industriais, de maneira evidente, são as que representam os maiores riscos de contaminação nos recursos hídricos, solo e ar devido à variedade, periculosidade e concentração de produtos químicos que estas gerenciam, e também algumas práticas de deposição de resíduos e efluentes que empregam. Porém, um dos pontos fundamentais na contaminação de áreas está relacionado ao acesso à água subterrânea.

A potencial contaminação de águas subterrâneas é de vital interesse na região oeste do estado de São Paulo, pois, de acordo com Prandi et al. (1998) os poços tubulares profundos representam a sua principal fonte de abastecimento de água. Segundo os autores, a falta de programas de operação e manutenção dos poços em funcionamento bem como sobre os poços abandonados pode oferecer chances de possíveis contaminações que atingiriam diretamente a população que se servem destes. Os estudos de Silva et al. (2020) e Silva et al. (2020), constataram a proximidade de áreas contaminadas com poços de captação de água e cursos d'água em locais urbanos de elevada concentração populacional, dessa maneira, representando ameaça para o meio ambiente e a saúde humana. Essas informações trazem à tona a necessidade de se realizar diagnósticos sobre áreas contaminadas a fim de se poder colaborar com a elaboração de políticas públicas mais específicas.

De acordo com a lei estadual n. ${ }^{\circ} 13.577$ de 8 de julho de 2009, área contaminada, no Estado de São Paulo, é definida como área, terreno, local, instalação, edificação ou benfeitoria que contenha quantidades, ou concentrações de matéria em condições que causem ou possam causar danos à saúde humana, ao meio ambiente ou a outro bem a proteger. O acompanhamento e a gestão das áreas contaminadas no estado de São Paulo são realizados pela Companhia Ambiental do Estado de São Paulo (CETESB), a qual desde 2002 divulga anualmente listagem de áreas contaminadas em todo o território estadual. Conforme a lei estadual n. ${ }^{\circ}$ 13.577, o gerenciamento das áreas contaminadas deve ser realizado pela CETESB e é baseado no cadastrado dessas áreas. Tal cadastro correspondente ao conjunto de informações referentes aos empreendimentos e atividades que apresentam potencial de contaminação e às áreas suspeitas de contaminação e contaminadas, distribuídas em classes de acordo com a etapa do processo de identificação e remediação da contaminação em que se encontram.

A existência de uma área contaminada pode gerar problemas, como danos à saúde, comprometimento da qualidade dos recursos hídricos, restrições ao uso do solo e danos ao patrimônio público e privado, com a desvalorização das propriedades, além de danos ao meio ambiente (CETESB, 2017).

As bacias hidrográficas Aguapeí e Peixe, as quais correspondem às unidades de gerenciamento de recursos hídricos (UGRHI's) 20 e 21 estão situadas no oeste paulista. Essas bacias têm como principais atividades econômicas a pecuária extensiva e o cultivo da cana-de-açúcar, que são importantes para a economia da região (CBH-AP, 2014).

Os municípios contidos nessas bacias hidrográficas são parcialmente abastecidos pelas águas do Sistema Aquífero Bauru, considerado a maior unidade hidrogeológica em área exposta do estado de São Paulo. Esse aquífero comportar-se principalmente como livre e possui grande área de afloramento apresentando, assim, expressiva vulnerabilidade à contaminação antrópica, demonstrando os mais elevados valores de concentração de nitrato nas águas subterrâneas no estado de São Paulo (IG/CETESB/DAEE, 1997; Modesto et al., 2009; CETESB, 2012).

Dentro deste contexto, a pesquisa foi desenvolvida com o objetivo de realizar o levantamento das áreas contaminadas nas bacias hidrográficas do rio Aguapeí e do rio do Peixe, relacionando a mudança no número de casos ao longo do tempo, a identificação das principais atividades potencialmente contaminantes e os impactos que estas promoveram no meio físico. 


\section{Materiais e Métodos}

\section{1 Área de abrangência}

De acordo com CBH-AP (2014) a bacia hidrográfica do rio Aguapeí, possui uma área de $13.196 \mathrm{~km}^{2}$ e situa-se entre as coordenadas geográficas $20^{\circ} 25^{\prime}$ a $21^{\circ} 32^{\prime}$ latitude sul e $49^{\circ} 26^{\prime}$ à $51^{\circ} 50^{\prime}$ longitude oeste. Já a bacia hidrográfica do rio do Peixe, dispõe de uma área de $10.769 \mathrm{~km}^{2} \mathrm{e}$ localiza-se entre as coordenadas geográficas $21^{\circ} 32^{\prime}$ a $22^{\circ} 25^{\prime}$ latitude sul e $49^{\circ} 50^{\prime}$ a $52^{\circ} 05^{\prime}$ de longitude oeste, ambas localizadas no Planalto Ocidental paulista, região oeste do estado de São Paulo (Figura 1). Ambas as bacias hidrográficas abrangem um total de 90 municípios, sendo 59 com sede na própria UGRHI e 31 com sede em outras UGRHI's. As bacias hidrográficas dos rios Aguapeí e Peixe (BHAP) possuem como substrato geológico aflorante as rochas vulcânicas da Formação Serra Geral, as rochas sedimentares de idade mesozoica do Grupo Bauru e depósitos aluvionares de idade cenozoica (Montero \& Peixoto, 2013).

Figura 1. Localização das bacias hidrográficas do rio Aguapeí e do rio do Peixe.

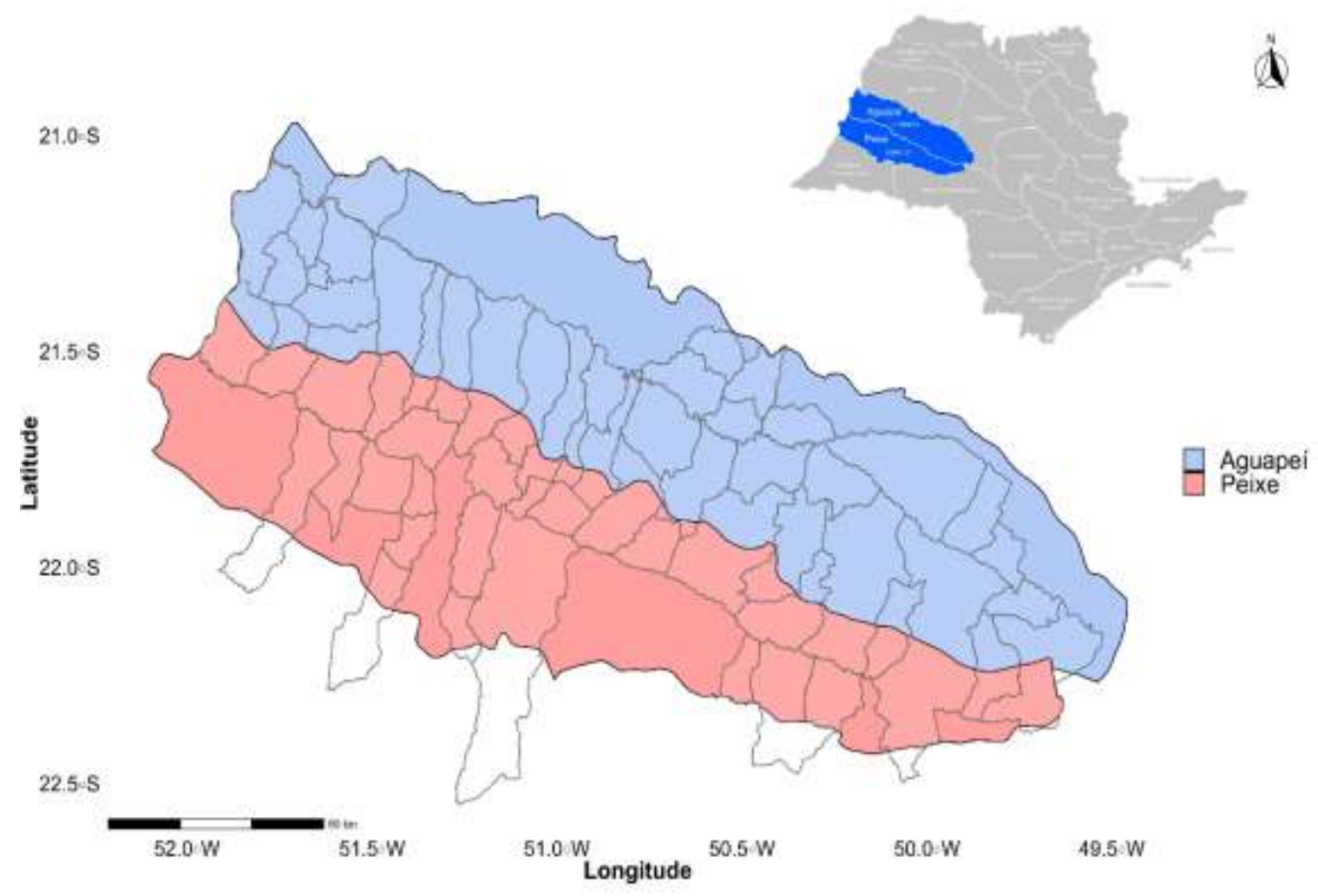

Fonte: CBH-AP (2014); Autores (2021).

\subsection{Coleta e análise de dados}

Foram utilizados dados de áreas contaminadas das BHAP, obtidos através dos Relatórios de Áreas Contaminadas e Reabilitadas do estado de São Paulo, publicados pela CETESB entre os anos de 2007 e 2019 (Tabela 1). Conjuntamente, foi extraído desses relatórios, informações sobre os tipos de atividades potencialmente contaminantes, os tipos de contaminantes e os meios negativamente impactados. Os mapas das áreas contaminadas foram elaborados com base nos arquivos shapefile obtidos a partir do Modelo Digital de Elevação (MDE) pelas imagens do Shuttle Radar Topography Mission (SRTM), extraídas do TOPODATA (Banco de Dados Geomorfométricos do Brasil). Estas informações foram processadas em um banco de dados com auxílio do software $R$. Por fim, os gráficos e mapas foram confeccionados com intuito de identificar quais são as 
áreas mais vulneráveis a contaminação, as principais atividades potencialmente contaminantes, os tipos de contaminantes mais recorrentes e os meios físicos mais impactados nas BHAP.

Tabela 1. Resumo do Relatórios de Áreas Contaminadas e Reabilitadas da BHAP.

\begin{tabular}{c|c|c|c|c}
\hline $\mathbf{A n o}$ & $\begin{array}{c}\text { Quantidade de } \\
\text { áreas contaminadas }\end{array}$ & $\begin{array}{c}\text { Quantidade de } \\
\text { atividades contaminantes }\end{array}$ & $\begin{array}{c}\text { Quantidade de } \\
\text { meios impactados }\end{array}$ & $\begin{array}{c}\text { Quantidade de } \\
\text { contaminantes }\end{array}$ \\
\hline $\mathbf{2 0 0 7}$ & 14 & 18 & 28 & 28 \\
\hline $\mathbf{2 0 0 8}$ & 16 & 24 & 34 & 34 \\
\hline $\mathbf{2 0 0 9}$ & 18 & 30 & 45 & 54 \\
\hline $\mathbf{2 0 1 0}$ & 22 & 36 & 50 & 65 \\
\hline $\mathbf{2 0 1 1}$ & 24 & 44 & 63 & 85 \\
\hline $\mathbf{2 0 1 2}$ & 25 & 49 & 71 & 87 \\
\hline $\mathbf{2 0 1 3}$ & 25 & 49 & 71 & 90 \\
\hline $\mathbf{2 0 1 4}$ & 26 & 52 & 76 & 95 \\
\hline $\mathbf{2 0 1 5}$ & 26 & 52 & 76 & 95 \\
\hline $\mathbf{2 0 1 6}$ & 26 & 54 & 81 & 98 \\
\hline $\mathbf{2 0 1 7}$ & 26 & 55 & 82 & 130 \\
\hline $\mathbf{2 0 1 8}$ & 26 & 55 & 83 & 136 \\
\hline $\mathbf{2 0 1 9}$ & 26 & 56 & 80 & \\
\hline
\end{tabular}

Fonte: CETESB (2019).

Buscando a identificação de padrões que pudessem ter relação na distribuição das áreas contaminadas foi estudada a correlação entre a quantidade de contaminantes encontrado nestas áreas e parâmetros socioeconômicos dos respectivos municípios pertencentes às BHAP. Para isso foram obtidos para cada município os dados de população, área em $\mathrm{km}^{2}$ e Índice de Desenvolvimento Humano Municipal (IDHM) disponibilizados pelo Instituto Brasileiro de Estatística e Geografia (IBGE, 2020). A partir desses dados, foi calculado o coeficiente de correlação de Pearson (r), também chamado de correlação linear de Pearson, o qual fora escolhido para verificar a associação entre os parâmetros, visto que, este método demonstra o grau de relação entre duas variáveis quantitativas e exprime a intensidade de uma relação linear entre dois conjuntos de dados (Figueiredo Filho \& Silva Junior, 2009).

A correlação de Pearson, representado pela letra "r", foi submetida a classificação de Bonfim e Januário (2018), que estabelece classes para o Coeficiente de Correlação de acordo com os valores adimensionais situados entre -1 e 1 (Tabela 2). Em que, $r>0.9$ significa uma correlação muito forte entre às duas variáveis e $r<-0.9$ expressa uma correlação inversa muito forte. Isto é, se uma variável aumenta, a outra diminui.

Em um segundo momento, a fim de procurar identificar outras possíveis combinações, os valores dos dados socioeconômicos (área, população e IDHM) foram multiplicados entre si e seus produtos (área x população e população x IDHM) foram submetidos à análise de correlação com o número de total de contaminantes das áreas contaminadas. Ressaltando que, o uso das variáveis socioeconômicas de forma isolada pouco representaria o conceito de urbanização das cidades, que segundo Paviani (1996), este conceito está relacionado com o crescimento populacional e físico-estrutural das cidades, conjuntamente com as transformações ou mudanças sociais e econômicas. 
Tabela 2. Interpretação do coeficiente de correlação de Pearson.

\begin{tabular}{c|c|c|c}
\hline \multicolumn{5}{c}{ Coeficiente de correlação Linear de Pearson } \\
\hline Correlação muito forte & $>0.9$ & Correlação Inversa muito forte & $<-0,9$ \\
\hline Correlação forte & 0,7 a 0,9 & Correlação inversa forte & $-0,9$ a 0,7 \\
\hline Correlação moderada & 0,5 a 0,7 & Correlação inversa moderada & $-0,7$ a $-0,5$ \\
\hline Correlação fraca & 0,3 a 0,5 & Correlação Inversa fraca & $-0,5$ a $-0,3$ \\
\hline Correlação desprezível & 0 a 0,3 & Correlação inversa desprezível & $-0,3$ a 0 \\
\hline
\end{tabular}

Fonte: Adaptado de Bonfim e Januário (2018).

O coeficiente de correlação de Pearson é expresso pela equação seguinte:

$$
r=\frac{1}{n-1}\left(\frac{x(x i-X)(y i-Y)}{\sqrt{\Sigma(x i-X)^{2}(y i-Y)^{2}}}\right) \quad i=1 \ldots n
$$

Em que $x i$ e $y i$ correspondem os valores médios de ambas as variáveis na observação $i$; $X$ e $Y$ são as médias aritméticas de ambas as variáveis.

Os dados também foram submetidos à Regressão local (LOESS) a qual foi conduzida por se tratar de um método nãoparamétrico que utiliza suavização de dispersão (smoothing) para ajustar curvas e superfícies. Segundo Marquetti \& Viali (2004), uma análise não-paramétrica, em contraposição com o método paramétrico, estima uma função média sem direcionar a uma forma funcional previamente estabelecida, permitindo que os "dados falem por si próprios". Deste modo, a LOESS consiste em observar os possíveis desvios na linearidade, permitindo facilmente a percepção de relações não-lineares.

\section{Resultados e Discussão}

\subsection{Atividades contaminantes nas bacias hidrográficas dos rios Aguapeí e Peixe}

A quantidade de atividades potencialmente contaminantes nas BHAP tem crescido nos últimos 13 anos (Figura 2), passando de um total de 18 atividades no ano de 2007 para 56 em 2019, dessa maneira representando um aumento de $210 \%$ na constatação de novas fontes contaminantes. Essa elevação não está ligada somente ao aumento das atividades poluidoras nas bacias hidrográficas, mas também, segundo CETESB (2019), está relacionado ao aumento das fiscalizações e consequente identificação das fontes potencialmente contaminantes.

Os resultados demonstraram que a maioria das contaminações foi proveniente de postos de combustíveis, correspondendo a $83 \%$ e $91 \%$ do total das atividades contaminantes nos anos de 2007 e 2019, respectivamente, demonstrando dessa maneira que os postos de combustíveis são a principal atividade geradora de contaminação nas BHAP. Esses dados estão de acordo com CETESB (2019), na qual esse tipo de fonte de contaminação também é encontrado nas outras bacias hidrográficas do Estado de São Paulo, respaldando também os trabalhos de Lino e Aquino (2017) e Spagnoli (2019).

As causas das contaminações por esse tipo de atividade estão ligadas principalmente ao vazamento acidental de derivados de petróleo para o meio ambiente. De acordo com Carneiro et al. (2020) os vazamentos e consequente contaminação gerada pelos postos de combustíveis tem potencial suficiente para inviabilizar o consumo de milhões de metros cúbicos de água subterrânea. Isto pode ocorrer em virtude da grande quantidade de combustível estocada pelos postos, por sua ampla distribuição por todas as duas bacias hidrográficas, mas também em função da idade dos empreendimentos. Segundo Corseuil 
\& Marins (1997) na década de 70 houve um grande aumento do número de postos de gasolina no país, é de se supor que a vida útil dos tanques de armazenamento já esteja próxima do final.

Figura 2. Evolução do número de atividades contaminantes nas BHAP entre os anos de 2007 e 2019.

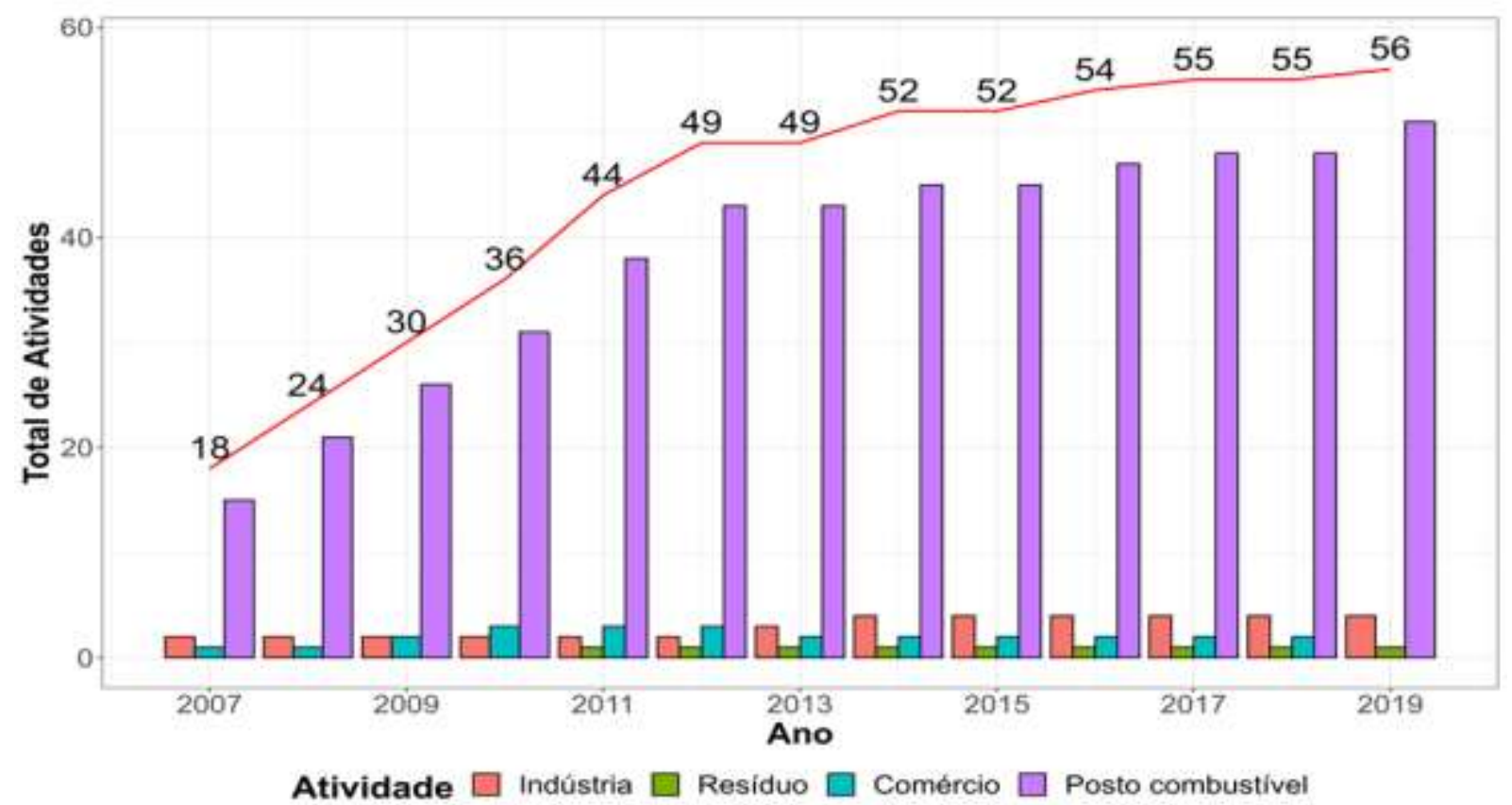

Fonte: Autores (2021).

De acordo com Gouveia e Nardocci (2007) dados da Agência Nacional do Petróleo, Gás Natural e Bicombustíveis (ANP) indicam que São Paulo é o Estado que possui o maior número de postos de combustíveis no país e se localizam, principalmente, nos grandes centros urbanos. Deste modo, as práticas preventivas contra acidentes ambientais necessitam de maior eficiência em razão do grande número de pessoas possivelmente expostas.

O número de áreas contaminadas a partir das atividades industrial, comercial e de produção de resíduos nas BHAP foi praticamente constante ao longo do tempo.

\subsection{Número de áreas contaminadas nas Bacias hidrográficas dos rios Aguapeí e Peixe}

Dos 90 munícipios inseridos nas BHAP, 26 foram identificados com pelo menos uma área contaminada (Figura 3), entretanto, a maioria das ocorrências foram concentradas nas cidades de Marília, Adamantina, Dracena e Presidente Prudente representando $47,8 \%$ do total de áreas contaminadas. Marília e Presidente Prudente por sua vez são os municípios mais populosos nas duas bacias hidrográficas.

Pode se observar a maior concentração de áreas contaminadas junto à linha do divisor de águas entre as duas bacias hidrográficas. Isso se deve ao fato que a maior parte das cidades nessa região foram instaladas ao longo do processo de colonização nas regiões mais altas do relevo em virtude da construção da estrada de ferro que garantiu a expansão territorial no começo do século 20.

Os principais contaminantes identificados foram solventes aromáticos, combustíveis líquidos, hidrocarbonetos policíclicos aromáticos (PAH), metais pesados, hidrocarbonetos totais de petróleo (TPH) e outros contaminantes, salientando que os mesmos foram reincidentes ao longo do período analisado. Os solventes aromáticos são constituídos pelo benzeno, tolueno, etilbenzeno e xilenos (BTEX) e os PAHs são substâncias tóxicas e cancerígenas, ambas consideradas como os 
principais contaminantes capazes de impactar o meio ambiente em casos de derramamentos de combustíveis (Rego \& Netto, 2007; Wu et al., 2010; Panagos et al., 2013; Li et al., 2020). Já o TPH, composto por uma grande variedade de produtos químicos, é considerado um indicador de presença de hidrocarbonetos no solo, água e efluentes (Forte et al., 2007; Alharbi et al., 2018). Diferente dos contaminantes orgânicos, os metais pesados têm elevados níveis de reatividade e bioacumulação, portanto, mesmo removendo a fonte contaminante, as concentraç̃̃es de metais no solo persistem por muito tempo (Guo et al., 2006). Os solventes aromáticos e os PAHs constituíram os contaminantes mais recorrentes dentro do período de estudo, representando $41,4 \%$ e $26,5 \%$, respectivamente.

Figura 3. Identificação das áreas contaminadas nas bacias hidrográficas dos rios Aguapeí e Peixe.

$$
\text { A) } 2007 \text {; B) 2011; C) 2015; D) } 2019 .
$$

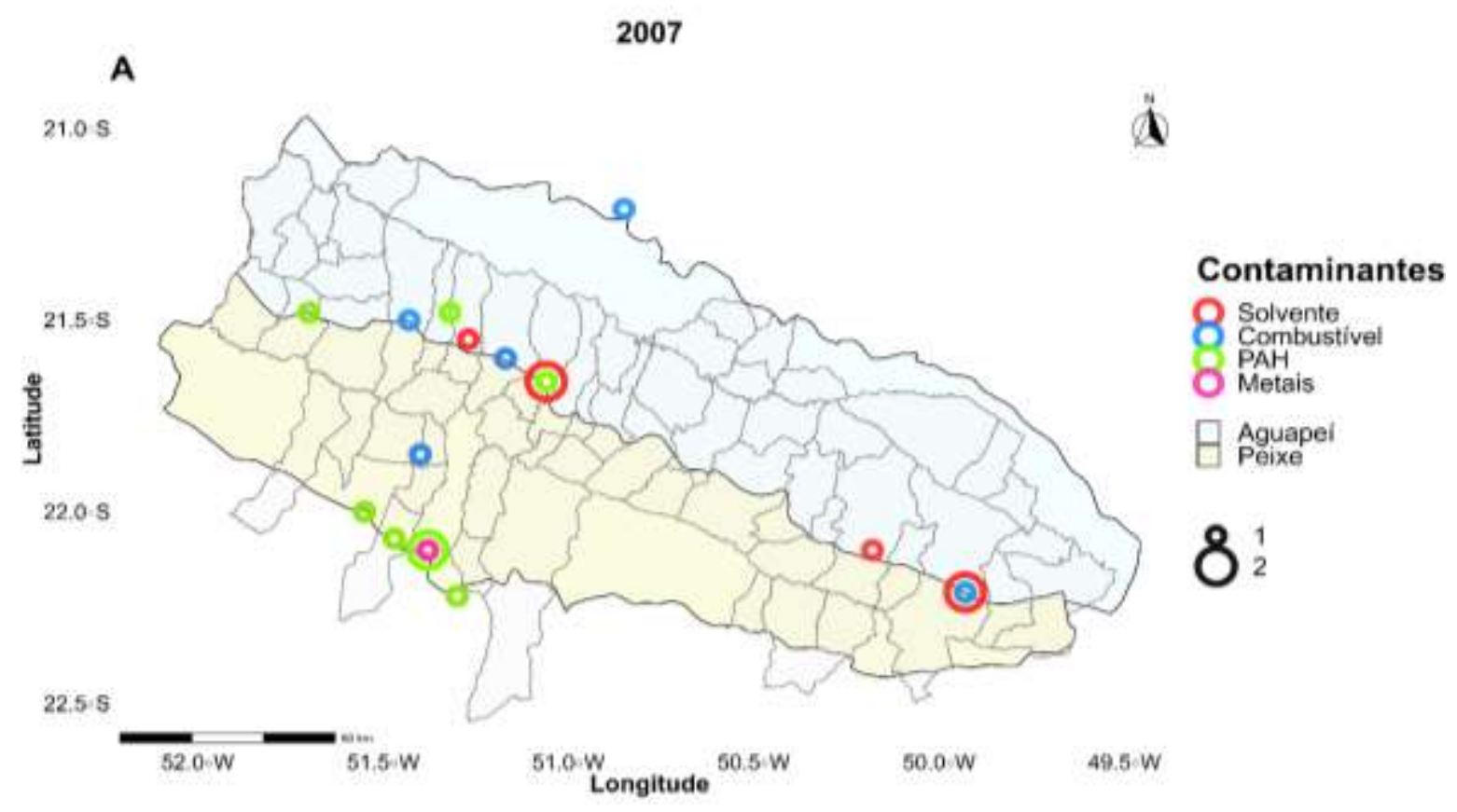


B

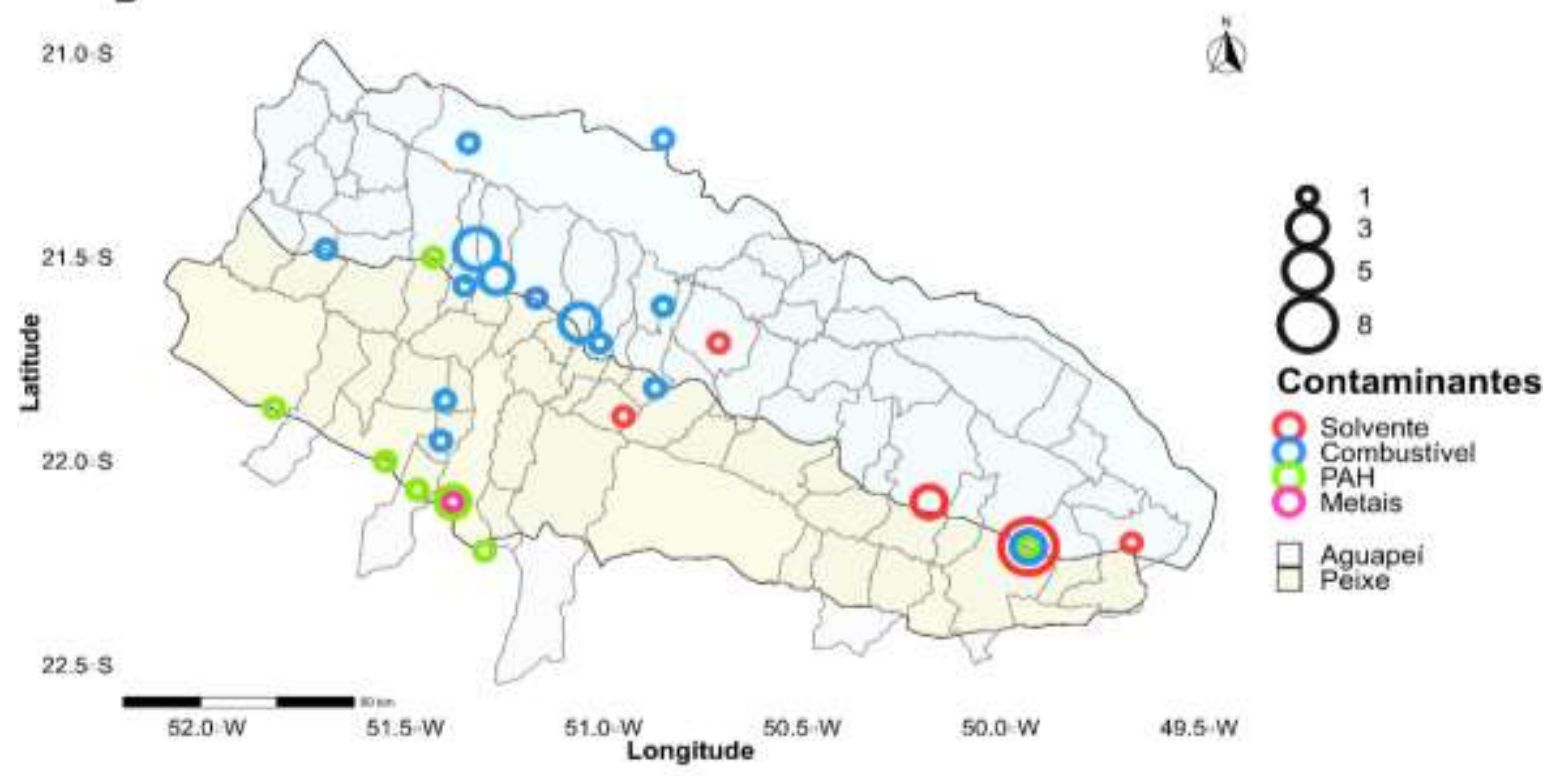

C

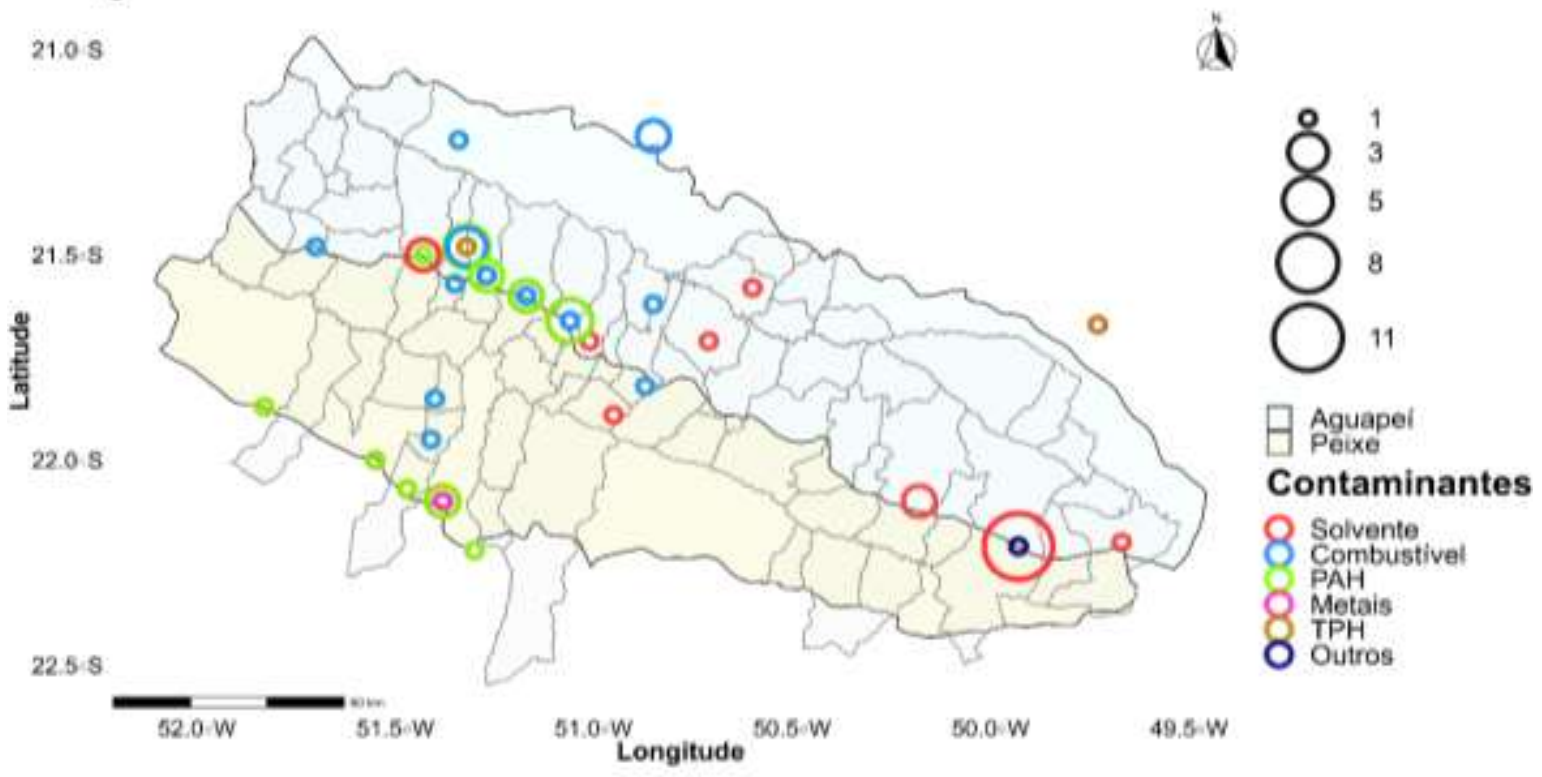




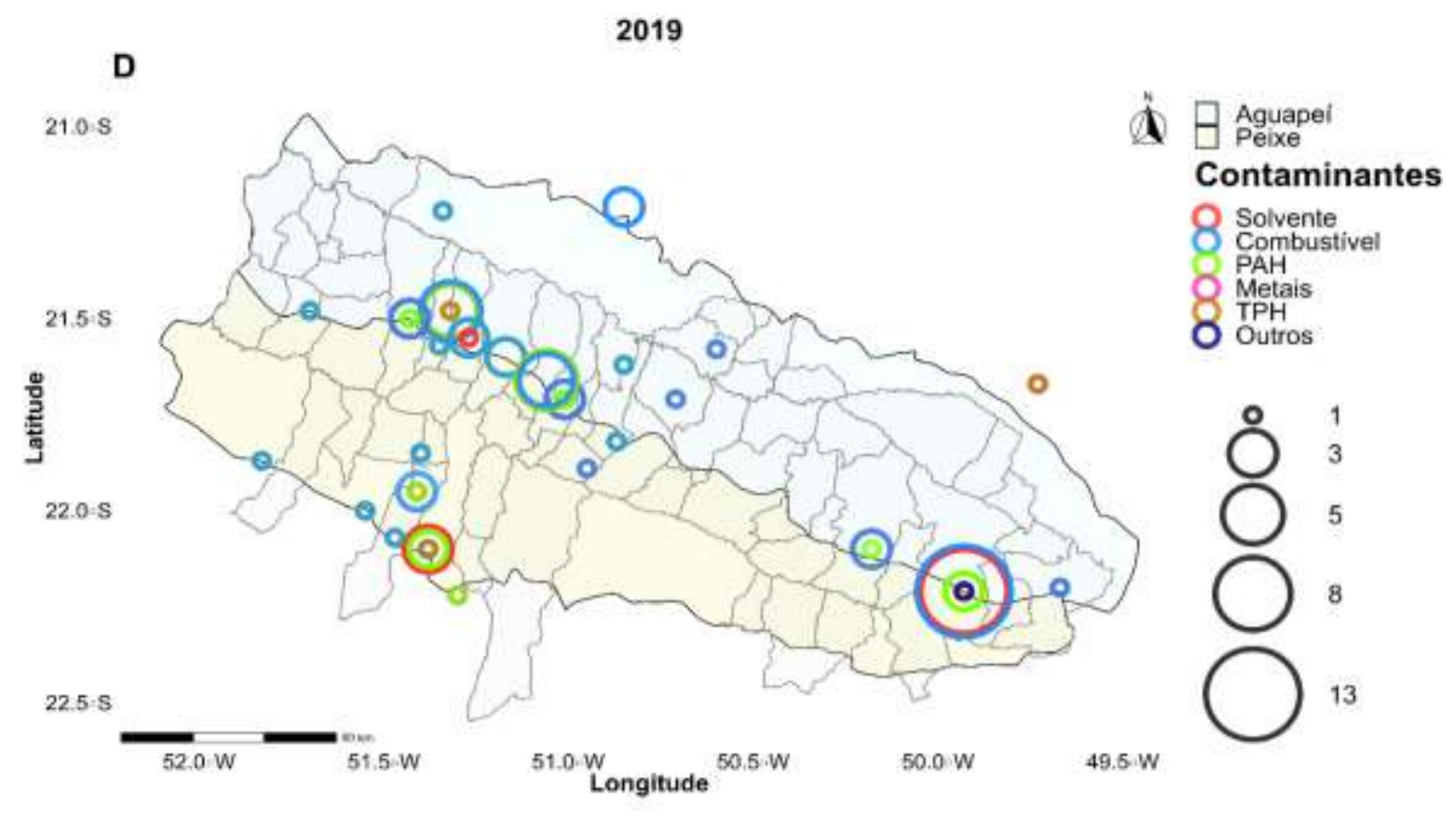

Fonte: Autores (2021)

No ano de 2007 (Figura 3A), foram identificados 4 tipos de contaminantes: metais pesados, combustíveis líquidos, solventes aromáticos e PAH distribuídos em 16 cidades ao longo da BHAP. Já no ano de 2011 (Figura 3B), os mesmos tipos de contaminantes também foram encontrados, entretanto, distribuídos em 24 municípios e em quantidades superiores ao ano de 2007, concentrados principalmente em Marília, Adamantina e Dracena. A concentração dos contaminantes é caracterizada pela variação no tamanho dos círculos, no qual um círculo maior representa um maior número de contaminante em uma determinada área.

Nos anos de 2015 (Figura 3C) e 2019 (Figura 3D) foram relatados dois (2) novos tipos de contaminantes identificados (TPH e outros contaminantes), houve também aumento na quantidade de contaminantes e no número de áreas contaminadas, totalizando os 26 municípios com locais contaminados encontrados neste estudo. No período entre 2015 e 2019 ocorreu um aumento de $69 \%$ na quantidade de contaminantes, contudo, no ano de 2015 os solventes aromáticos foram os contaminantes mais relatados, representando 46,31\%, seguido dos PAH com 28,42\% e no ano de 2019 , os contaminantes mais incidentes foram combustíveis líquidos $(36,74 \%)$ e solventes aromáticos $(33,82 \%)$. Resultados semelhantes, com áreas contaminadas oriundas de postos de combustíveis, foram descritos por Rego \& Netto (2007) para o município do Rio de Janeiro, Lima et al. (2017) para o município de Cuiabá e Silva et al. (2020) para o município de Jundiaí.

\subsection{Meios impactados nas bacias hidrográficas dos rios Aguapeí e Peixe}

Os relatórios de Áreas Contaminadas e Reabilitadas emitidos pela CETESB apontaram impactos negativos causados pela presença de contaminantes no ar, solo superficial, subsolo e na água subterrânea. As águas subterrâneas e os subsolos foram os meios mais impactados pelos contaminantes nas BHAP, em tese representando conjuntamente $95 \%$ dos meios impactados (Figura 4).

Em comparação com 2007 o ano de 2019 demonstrou um aumento de cerca de 200\% na incidência de contaminação das águas subterrâneas e dos subsolos na área de estudo, respaldando os resultados encontrados por Carneiro et al. (2020). Os baixos valores de contaminação no ar e no solo superficial estão relacionados com as atividades industriais e áreas de 
destinação de resíduos sólidos, que foram menos contabilizadas nos Relatórios de Áreas Contaminadas e Reabilitadas utilizados neste estudo.

Figura 4. Número de meios impactados nas bacias hidrográficas dos rios Aguapeí e Peixe no período de 2007 a 2019.

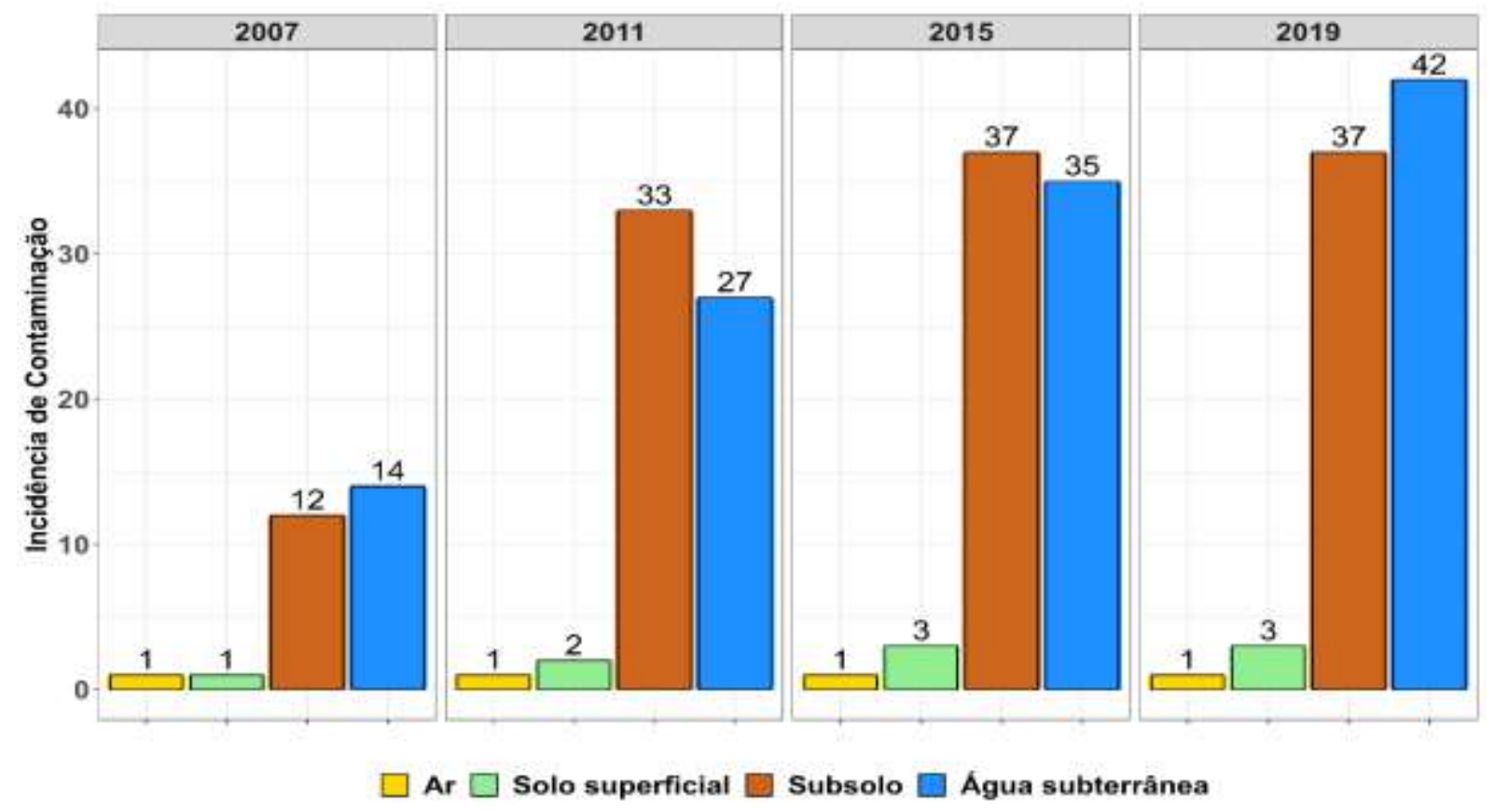

Fonte: Autores (2021).

Segundo CETESB (2019), a maioria das contaminações no subsolo e águas subterrâneas ocorrem por via de vazamentos ou infiltrações de produtos oriundos de postos de combustíveis. Quando ocorre um derramamento, o combustível líquido irá infiltrar primeiro nos poros preenchidos com ar, formando uma fina película, em seguida a contaminação pode ocorrer através do processo de infiltração, lixiviação, pela variação do nível freático e também por meio das forças gravitacionais, que direcionam o fluxo para as porções mais profundas do solo, com possibilidade de alcance às águas subterrâneas (Baird, 2002; Santos, 2009; Logeshwaran et al., 2018).

De acordo com Hirata e Ferreira (2001) e Li et al. (2020), existe um alto grau de correlação entre a idade dos tanques de armazenamento e uma maior probabilidade de vazamentos. A Resolução n. ${ }^{\circ}$ 273/00 do Conselho Nacional do Meio Ambiente (CONAMA) afirma que todo sistema de estocagem de derivados de petróleo e outros combustíveis pode contaminar tanto águas superficiais, quanto as subterrâneas contidas nos aquíferos, classificando-os assim como, potenciais poluidores e geradores de acidentes ambientais (Barros et al., 2016).

Segundo Logeshwaran et al. (2018) a contaminação ambiental proveniente de hidrocarbonetos de petróleo (solventes, óleos, combustíveis e derivados) já é uma questão global e as práticas de remediação ambiental nesses locais devem ser priorizadas. Importante ressaltar que os postos de combustíveis e derivados são uma das principais fontes de contaminação dos subsolos e águas subterrâneas também em outros países. Resultados similares foram encontrados por Panagos et al. (2013) nos países do continente Europeu e Alharbi et al. (2018) na Arábia Saudita.

\subsection{Correlação entre total de contaminantes e os dados socioeconômicos}

Com base no período de 13 anos analisados, a correlação de Pearson mais consistente foi encontrada entre o total de contaminantes e o produto (área x população) que apresentou um valor de $r=0.76$, significando uma correlação forte (Figura 
5). Isto indica, neste caso, que o maior número de contaminantes, estatisticamente, encontra-se nas áreas mais urbanizadas (que possuem a maior área e o maior número de habitantes). A exemplo, a cidade de Marília que contabilizou o maior número de contaminantes. Semelhantemente, as cidades que relataram os menores totais de contaminantes também apresentaram valores reduzidos no produto (área x população), ou seja, as cidades menores. Já a correlação entre o produto (população x IDHM) e o número de contaminantes foi menor $(r=0.66)$, indicando uma correlação moderada.

Pelo fato das bacias hidrográficas abordadas neste estudo não abrigarem grandes centros urbanos, as cidades de médio porte são consideradas as mais importantes do oeste paulista, decorrente da influência regional que possuem em função das atividades industriais mais desenvolvidas (Gomes, 2007). As cidades de Marília, Presidente Prudente, Lins, Garça, Adamantina e Dracena apresentaram os maiores valores em relação à área, população e IDHM, sendo considerados os centros urbanos potencialmente mais desenvolvidos das BHAP.

Figura 5. Correlação entre o número de áreas contaminadas (eixo y) e os dados socioeconômicos Área x População e População x IDHM (eixo x em forma exponencial). A linha vermelha representa a regressão linear de Pearson e a linha azul, a regressão local (LOESS).
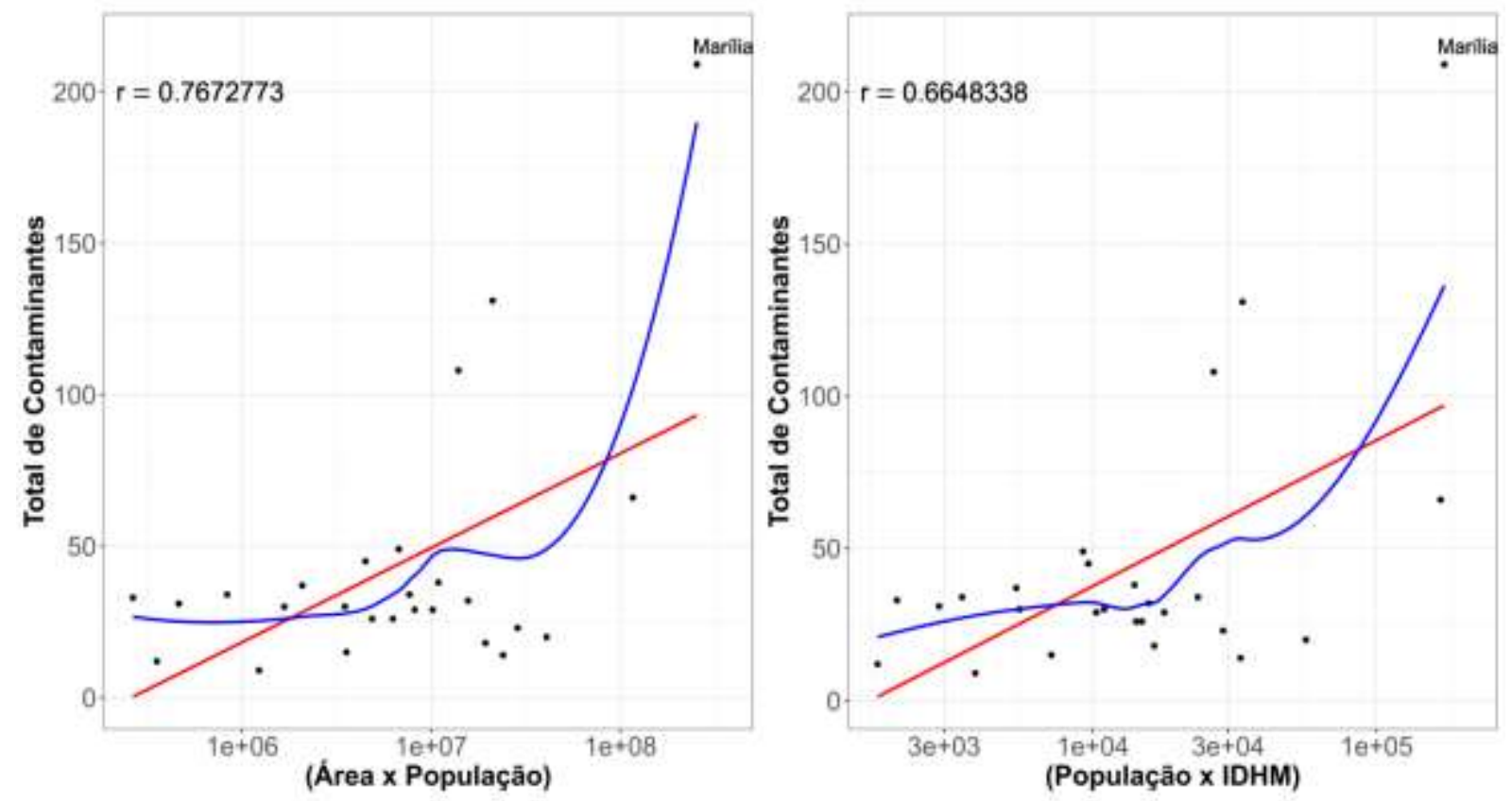

Fonte: Autores (2021).

Salientando que apesar de constarem nos relatórios de áreas contaminadas das BHAP, os municípios de Presidente Prudente e Lins não detêm áreas inteiramente nas bacias hidrográficas em estudo, visto que os valores de contaminação desses dois municípios são divididos em outros relatórios de áreas contaminadas não contabilizadas neste trabalho.

Ao estimar a LOESS, sugeriu que a relação entre os números totais de contaminantes e os produtos extraídos dos dados socioeconômicos dos municípios pertencentes às BHAP, em ambas as análises, puderam ser melhor descritos adequadamente usando um encaixe não linear para os números de contaminantes inferior à 50. Entretanto, para as cidades de médio porte, que apresentaram valores superiores de contaminantes, a correlação linear de Pearson foi mais satisfatória, indicando uma relação positiva entre o maior número de contaminantes e as cidades potencialmente mais desenvolvidas.

Utilizando a correlação de Pearson, Souza \& Gastaldini (2014) relataram que os piores resultados de degradação ambiental na bacia hidrográfica do Rio Vacacaí - Mirim, foram encontrados nas áreas que apresentaram o maior percentual de 
urbanização e o maior número de habitantes. Da mesma forma, Freire e Castro (2014) demonstraram que o agravo nos índices de qualidade da água está correlacionado com alto grau de atividade antropogênica na bacia hidrográfica do rio Itapemirim.

Em suma, os municípios de Marília, Adamantina, Dracena e Presidente Prudente foram identificados como as áreas mais vulneráveis a contaminação em decorrência dos maiores parâmetros área, população e IDHM.

\section{Conclusão}

Com base nos Relatórios de Áreas Contaminadas e Reabilitadas do Estado de São Paulo emitidos pela CETESB, verificou-se que o número de áreas contaminadas nas bacias hidrográficas dos rios Aguapeí e Peixe aumentaram expressivamente ao longo dos 13 anos de estudo.

A principal atividade responsável por contaminações na região de estudo foram os postos de combustíveis e derivados, dos quais os solventes aromáticos e os PAHs foram os contaminantes mais recorrentes.

Os relatórios de áreas contaminadas do Estado de São Paulo publicados pela CETESB configuram-se como uma importante ferramenta no gerenciamento e reabilitação das áreas contaminadas no âmbito estadual. Apesar da publicação dos relatórios ser anual e sistemática, a divulgação das informações de forma conjunta pode colaborar para expressar a potencial importância das áreas contaminadas nas diferentes regiões do estado de São Paulo. Dessa maneira, a metodologia proposta no trabalho, que visou a elaboração do mapeamento cartográfico evolucional dos locais contaminados nas bacias hidrográficas dos rios Aguapeí e Peixe, pode contribuir com esse processo.

\section{Referências}

Alharbi, B. H., Pasha, M. J, Alhudhodi, A. H. \& Alduwais, K. (2018). Assessment of soil contamination caused by underground fuel leakage from selected gas stations in Riyadh, Saudi Arabia. Soil and Sediment Contamination: An International Journal. 27(8), 674-691.

Baird, C. (2002). Química Ambiental, (2a ed.). Bookman.

Barros, C. G. D., Rosa, A. L. D., Silva, J. L. S., Oliveira, G. A. \& Teixeira, R. B. S. S. (2016). Superfície Potenciométrica e Possíveis Fontes de Contaminação do Aquífero Parecis no Município de Vilhena-RO, BR. Revista Monografias Ambientais. 15, 74-84.

Bonfim, O. E. T. \& Januário, I. R. (2018). Correlação entre a precipitação e temperatura média do ar sobre o número de registros de casos de dengue para cidades de Maceió, João Pessoa e Salvador. Revista de Geografia (Recife), 35(5).

Braun, A. B., Trentin, A. W. S, Visentin, C. \& Thomé, A. (2020). Relevance of sustainable remediation to contaminated sites manage in developed and developing countries: Case of Brasil. Land Use Policy, 94, 1-11.

Carneiro, G. C. A., Dias, D. A. F., Fonseca, E. R. \& Gonçalves, J. A. C. (2020). Contaminação das águas subterrâneas por compostos orgânicos na Bacia hidrográfica do rio das velhas, estado de Minas Gerais, Brasil. Research, Society and Development. 9, 1-19. 10.33448/rsd-9i10.8536.

CBH-AP - Comitê das Bacias Hidrográficas dos Rios Aguapeí e Peixe. (2014). Relatório de Situação dos Recursos Hídricos 2014. Marília, 51p.

CETESB - Companhia Ambiental do Estado de São Paulo. (2012). Qualidade das águas subterrâneas do estado de São Paulo 2010-2012. 242p.

CETESB - Companhia Ambiental do Estado de São Paulo. (2015). Texto explicativo Relatório de contaminadas e reabilitadas no Estado de São Paulo. 21p. https://cetesb.sp.gov.br/areas-contaminadas/wp-content/uploads/sites/17/2013/11/Texto-explicativo.pdf.

CETESB - Companhia Ambiental do Estado de São Paulo. (2017). Texto explicativo Relatório de contaminadas e reabilitadas no Estado de São Paulo. 21p. https://cetesb.sp.gov.br/areas-contaminadas/wp-content/uploads/sites/17/2018/01/Texto-explicativo.pdf.

CETESB - Companhia Ambiental do Estado de São Paulo. (2019). Relação de áreas contaminas e reabilitadas no estado de São Paulo. São Paulo. https://cetesb.sp.gov.br/areas-contaminadas/relacao-de-areas-contaminadas/.

Corseuil, H. X. \& Marins, M. D. M. (1997). Contaminação de águas subterrâneas por derramamentos de gasolina: O problema é grave? Revista Engenharia Sanitária e Ambiental, 2, 50-54.

Figueiredo Filho, D. B. \& Silva Junior, J. A. (2009). Desvendando os Mistérios do Coeficiente de Correlação de Pearson (r). Revista Política Hoje, 18(1), 115146.

Forte, E. J., Azevedo, M. S., Oliveira, R. C. \& Almeida, R. (2007). Contaminação de aquífero por hidrocarbonetos: estudo de caso na Vila Tupi, Porto Velho Rondônia. Química Nova.30, 1539-1544. 10.1590/S0100-40422007000700008.

Freire, A. P. \& Castro, E. C. Análise da Correlação do uso e Ocupação do Solo e da Qualidade da Água. Revista Brasileira de Recursos Hídricos, v.19, n.1, 2014, pp.41-49. https://doi.org/10.21168/rbrh.v19n1.p41 
Gomes, M. T. S. (2007). O processo de reestruturação produtiva em cidades médias do oeste paulista: Araçatuba, Birigui, Marília, Presidente Prudente e São José do Rio Preto. 295f. Tese (Doutorado em Geografia Humana). Universidade de São Paulo.

Gouveia, J. L. N. \& Nardocci, A. C. (2007). Acidentes em Postos e Sistemas Retalhistas de Combustíveis: Subsídio para a Vigilância em Saúde Ambiental. Eng. Sanit. Ambient. 12(3), 317-324.

Günther, W. M. R. (2006) Áreas contaminadas no contexto da gestão urbana. São Paulo em Perspectiva. 20(2), 105-117.

Guo, G. L., Zhou, Q. X., Koval, P. V. \& Belogolova, G. A. (2006) Speciation distribution of Cd, Pb, Cu, and Zn in contaminated Phaeozem in north-east China using single and sequential extraction procedures. Australian Journal of Soil Research, 44(2), 135-142. 10.1071/SR05093

Hirata, C. A. \& Ferreira, L. M. R. (2001). Os Aquíferos da Bacia Hidrográfica do Alto Tietê: Disponibilidade Hídrica e Vulnerabilidade à Poluição. Revista Brasileira de Geociências. 31, 43-50.

IBGE - Instituto Brasileiro de Estatística e Geografia. Cidades e Estados. (2020). https://www.ibge.gov.br/cidades-e-estados/ .

Instituto Geológico (IG), Companhia de Tecnologia de Saneamento Ambiental (CETESB), Departamento de Águas e Energia Elétrica (DAEE) (1997). Mapeamento da vulnerabilidade e risco de poluição das águas subterrâneas no Estado de São Paulo. IG, CETESB, DAEE.

Li, X., Wu, H. \& Qian, H. (2020). Groundwater contamination risk assessment using intrinsic vulnerability, pollution loading and groundwater value: a case study in Yinchuan plain, China. Environmental Science and Pollution Research. 27, 45591-45604.

Lima, S. D., Oliveira, A. F., Golin, R., Caixeta, D. S., Lima, Z. M. \& Morais, E. B. (2017). Gerenciamento de áreas contaminadas por postos de combustíveis em Cuiabá, Mato Grosso, Brasil. Ambiente \& Água. 12(2), 299-315. 10.4136/1980-993X

Lino, J. S. \& Aquino, A. R. (2017). Análise da Evolução das Áreas Contaminadas no Município de São Paulo, VII Congresso Brasileiro de Gestão Ambiental. Campo Grande.

Logeshwaran, P., Megharaj, M., Chadalavada, S., Bowman, M. \& Naidu, R. (2018). Petroleum hydrocarbons (PH) in groundwater aquifers: An overview of environmental fate, toxicity, microbial degradation and risk-based remediation approaches. Environmental Technology \& Innovation, 10, 175-193.

Marques, M. N., Cotrim, M. B., Pires, M. A. F. \& Beltrame Filho, O. (2007). Avaliação do impacto da agricultura em áreas de proteção ambiental, pertencentes à Bacia hidrográfica do Rio Ribeira de Iguape, São Paulo. Química Nova, 30(5), 1171-1178.

Marquetti, A. \& Vialli, L. (2004) Princípios e aplicações de regressão local. Análise Econômica, 22(42), 252-277.

Modesto, R. P., Dias, C. L., Toffoli, F. F. \& Vale, F. R. C. (2009). Evolução das concentrações de nitrato no Sistema Aquífero Bauru no Estado de São Paulo 1992 a 2007. In: ABAS, Congresso Internacional De Meio Ambiente Subterrâneo, 1, Anais, 11 p.

Montero, R. C. \& Peixoto, A. S. P. (2013). Vulnerabilidade e perigo de contaminação dos aquíferos no alto Aguapeí e alto Peixe, SP. Ciência \& Engenharia, $22(1), 115-124$.

Panagos, P., Van Liedekerke, M., Yigini, Y. \& Montanarella, L. (2013). Contaminated sites in Europe: Review of the current situation based on data collected through a European network. Journal of Environmental and Public Health. 2013, 1-11.

Paviani, A. (1996). Urbanização: impactos ambientais da população. Revista Bioética, 4(2), 1-6.

Pinheiro, N. C. A. \& Mochel, F. R. (2018). Diagnóstico de áreas contaminadas pela disposição final de resíduos sólidos no município de Paço do Lumiar (MA). Eng.Sanit.Ambient, 23(6), 1173-1184. 10.1590/S1413-41522018173619

Prandi, E. C., Polegato, J. C., Oliveira Filho, J. A., Sabbag, E. G. \& Lobo, G. A. (1998). Águas subterrâneas: Fonte de abastecimento para a área do comitê dos rios Aguapeí e Peixe. Águas Subterrâneas, (1). São Paulo: ABAS, 8 p.

Rego, E. C. P \& Netto, A. D. P. (2007). PAHs and BTEX in ground water of gasoline stations from Rio de Janeiro City, Brazil. Bulletin of environmental contamination and toxicology, 79(6), 660-664.

Sánchez, L. E. (2001) Desengenharia: o passivo ambiental na desativação de empreendimentos industriais. Edusp, Fapesp, 256p.

Santos, D. A. R. (2009). Análise do monitoramento ambiental e da delimitação das plumas de contaminantes provenientes de vazamentos em postos de combustíveis: estudos de caso no Médio Vale do Paraíba.126 f. Dissertação (Mestrado em Engenharia Mecânica) - Faculdade de Engenharia do Campus de Guaratinguetá, Universidade Estadual Paulista, Guaratinguetá.

São Paulo. (2009). Lei $n^{\circ} 13.577$, de 8 de julho de 2009. Dispõe sobre diretrizes e procedimentos para a proteção da qualidade do solo e gerenciamento de áreas contaminadas. Assessoria Técnico-Legislativa. São Paulo.

Silva, F. P., Oliveira, E. A., Melilo, R. C. S., Oliveira, C. R. \& Gavioli, F. R. (2020). Identificação E Mapeamento De Áreas Contaminadas E Com Potencial De Contaminação De Recursos Hídricos No Município De Jundiaí. Divers@ Revista Eletrônica Interdisciplinar, 13(2), 186-197.

Silva, N. B. S, Borges, I. \& Vasconcelos, V. V. (2020). Análise do impacto potencial de áreas com contaminação de solos sobre cursos d'água e poços no município de São Paulo. Águas Subterrâneas, 34(3), 250-263.

Souza, M. M. D. \& Gastaldini, M. D. C. C. (2014). Avaliação da qualidade da água em bacias hidrográficas com diferentes impactos antrópicos. Engenharia Sanitária e Ambiental, 19(3), 263-274.

Spagnoli, M. V. (2019). Gerenciamento de áreas contaminadas e a gestão participativa das águas subterrâneas no Estado de São Paulo. 90f. Dissertação (mestrado em Recursos Hídricos), Faculdade de Engenharia, Universidade Estadual Paulista. Ilha Solteira. 
Research, Society and Development, v. 10, n. 8, e14710817264, 2021

(CC BY 4.0) | ISSN 2525-3409 | DOI: http://dx.doi.org/10.33448/rsd-v10i8.17264

Wu, B, Zhang, Y, Zhang, X. \& Cheng, S. (2010). Health risk from exposure of organic pollutants through drinking water consumption in Nanjing, China. Bull Environ Contam Toxicol, 84(1), 46-50. 2010. 10.1007/s00128-009-9900-8

Zasso, M. A. D. C., Ferreira, F., Lucchese, O., Attuati, M. A., Fernandes, S. B. V. \& Uhde, L. T. (2014). Meio ambiente e sustentabilidade. Ed. da Unijuí. $148 \mathrm{p}$ 